\title{
Multilinguales
}

1 | 2013

Pratiques littéraires, linguistiques, pédagogiques,

didactiques et médiations culturelles contemporaines

\section{La mort de Fama dans Les soleils des indépendances d'A. Kourouma: entre logique mythique et logique romanesque}

The death of Fama in The Suns of Independence by A. Kourouma:between

mythical logic and romantic logic

\section{Sabrina Zouagui}

\section{Q OpenEdition}

1 Journals

\section{Édition électronique}

URL : https://journals.openedition.org/multilinguales/2935

DOI : $10.4000 /$ multilinguales.2935

ISSN : 2335-1853

Éditeur

Université Abderrahmane Mira - Bejaia

Édition imprimée

Date de publication : 1 juin 2013

Pagination : 45-55

ISSN : 2335-1535

\section{Référence électronique}

Sabrina Zouagui, «La mort de Fama dans Les soleils des indépendances d'A. Kourouma: entre logique mythique et logique romanesque », Multilinguales [En ligne], 1 | 2013, mis en ligne le 01 juin 2013, consulté le 30 juin 2021. URL : http://journals.openedition.org/multilinguales/2935 ; DOI : https:// doi.org/10.4000/multilinguales.2935

Ce document a été généré automatiquement le 30 juin 2021.

\section{(c) $(9)$}

Multilinguales est mise à disposition selon les termes de la Licence Creative Commons Attribution Pas d'Utilisation Commerciale - Pas de Modification 4.0 International 


\title{
La mort de Fama dans Les soleils des indépendances d'A. Kourouma: entre logique mythique et logique romanesque
}

\author{
The death of Fama in The Suns of Independence by A. Kourouma:between \\ mythical logic and romantic logic
}

Sabrina Zouagui

1 Le roman subsaharien a construit un univers où se côtoient mythes, traditions et conceptions religieuses spécifiques au continent africain. Ce dernier est un immense réservoir d'éléments culturels étrangers au roman occidental. Il est donc à se demander comment ce genre littéraire moderne se réapproprie ces données culturelles et les réinvestit de son esthétique. Nous nous proposons de revisiter Les Soleils des Indépendances d'Ahmadou Kourouma ${ }^{2}$, à travers un épisode-clé qui clôt le roman: la mort de Fama. Notre intérêt a été suscité par l'incertitude qui plane sur cette mort, à mi-chemin entre mythification et démythification.

2 Notre attention a été retenue par l'absence de narration du rituel funéraire pour Fama, dans une société où ce rituel sacré est essentiel surtout pour les princes des grandes dynasties comme lui. Il nous importait alors de savoir pourquoi le personnage principal de ce roman en a été privé par le narrateur. Donc, la problématique de notre travail s'articule autour de la réponse à ces interrogations : quelle interprétation donner à cette «carence » remarquable sur le plan aussi bien narratif que culturel (mythique) ? De quelle façon la mort de Fama oscille-t-elle entre deux logiques, une logique mythique et une autre d'ordre romanesque?

3 La mort de Fama peut être en effet appréhendée à deux niveaux : la démystification de Fama suivie de sa mythification. Il est démythifié à travers sa déchéance sociale et la subversion du rituel funéraire de ses obsèques. Mais le texte finit par lui donner une dimension mythique. Nous disons qu'il $\mathrm{y}$ a re-mythification dans le sens où Fama 
acquiert la dimension de mythe (littéraire). Nous allons donc essentiellement étudier la façon dont le rite funéraire est textualisé d'abord pour les personnages de Koné Ibrahima, Balla et Lacina, ensuite pour Fama. Cette analyse servira, dans un premier temps, à mettre au jour le processus de démythification de la mort de Fama. Dans un second temps, elle nous permettra d'envisager le processus romanesque de transformation de ce personnage en mythe littéraire.

\section{Le rituel funéraire : fiction et réalité anthropologique}

4 Fama est un personnage controversé dans la société du roman. C'est un prince : le dernier descendant de la dynastie des Doumbouya. Mais il est dépossédé de son titre, ne peut assurer sa succession parce qu'il n'a pas eu d'enfant, ne vit que des offrandes distribuées lors des cérémonies. Aux funérailles de Koné Ibrahima, auxquelles il assistait au début du roman pour assurer sa subsistance, il est interpellé publiquement par « le très vieux griot » qui

mêlait aux éloges de l'enterré des allusions venimeuses (...). Et là, il se dévergonda et arriva au-delà de toute limite: des descendants de grands guerriers (c'était Fama!) vivaient de mensonges et de mendicité (c'était encore Fama), d'authentiques descendants de grands chefs (toujours Fama) avaient troqué la dignité contre les plumes de vautour et cherchaient le fumet d'un événement: naissances, mariages, décès, pour sauter de cérémonie en cérémonie. (...) Fama se leva, interrompit: Musulmans! pardon, musulmans! Ecoutez!... Impossible d'ajouter un mot. Une meute de chiens en rut : tous ces assis de damnés de Malinké se disant musulmans hurlèrent, se hérissèrent de crocs et d'injures. (17-18)

Rejeté par les siens parce qu'il n'honore pas son rang comme le veut la coutume, ce "grand prince » déchu continue, néanmoins, de brandir fièrement son titre de prince et de croire à la sacralité des ancêtres. Contrairement aux autres Malinkés (qu'il rejette aussi), il juge «l'ère des Indépendances », qui a vu les dynasties péricliter et son village, Togobala, sombrer dans la désolation, comme une «Bâtardise! Vraiment les soleils des Indépendances sont impropres aux grandes choses; ils n'ont pas seulement dévirilisé mais aussi démystifiél'Afrique » (143-144).

6 Cependant, au dernier chapitre du roman, Fama subit une transformation spectaculaire en se réhabilitant lui-même : il adopte une attitude conforme à son rang de prince, dédaigne toutes les richesses que «le parti unique» lui fait miroiter. Il n'a plus qu'un seul souhait: mourir sur la terre de ses ancêtres. Pour le réaliser, il bravera les autorités et franchira toutes les frontières interdites d'accès, pour mourir des suites de l'attaque d'« un caïman sacré» (194), à la fin du roman. Bien que "les deux infirmiers [qui] l'examinèrent dans l'ambulance (...) se récrièrent: "Allah tout puissant! Un caïman sacré n'attaque que lorsqu'il est dépêché par les mânes pour tuer (...) un grand sorcier ou un grand chef" » (194), Fama n’a pas eu droit à la cérémonie de ses funérailles, contrairement à plusieurs personnages secondaires du roman :

Fama avait fini, était fini. (...) Un Malinké était mort. Suivront les jours jusqu'au septième jour et les funérailles du septième jour, puis se succéderont les semaines et arrivera le quarantième jour et frapperont les funérailles du quarantième jour et... (195)

7 La valeur symbolique de ce préjudice, dans la fiction, est d'autant plus grande que ce rite funéraire a une forte dimension anthropologique dans la réalité des Malinkés. 


$$
\text { l'occasion de la mort de trois personnages du pays malinké, proches de Fama: Koné }
$$
Ibrahima, Lacina et Balla.

Pour Koné Ibrahima, la description se déploie selon la succession des étapes du rituel :

Des jours suivirent le jour des obsèques jusqu'au septième jour et les funérailles du septième jour se déroulèrent devant l'ombre, puis se succédèrent des semaines et arriva le quarantième jour, et les funérailles du quarantième jour ont été fêtées au pied de l'ombre accroupie. (10)

Pour le cousin Lacina, la narration du rituel, qui ouvre le chapitre 5 de la seconde partie du roman, se double d'une dimension pédagogique :

Pourquoi les Malinkés fêtent-ils les funérailles du quarantième jour d'un enterré ? Parce que quarante jours exactement après la sépulture les morts reçoivent l'arrivant mais ne lui cèdent une place et des bras hospitaliers que s'ils sont tous ivres de sang. (138)

Pour les « enivrer » et « préparer pour le cousin décédé un au-delà large » (138),

Fama, Balla, Diamourou (...) remontèrent aux grandes traditions et mirent à l'attache au milieu de la cour des Doumbouya, le matin des funérailles du quarantième jour, quatre bœufs. (...) Le sacrifice avait été accepté, totalement exaucé. Heureux étaient tous les morts, surtout les aïeux de Fama. Déjà l'enterré Lacina les avait rejoints. Jamais plus son double n'errera derrière les cases, ne hantera les rêves en quête de la place qui assure le calme de l'intérieur. (138-145)

Pour le féticheur Balla, le rituel est envisagé dans sa dimension fabuleuse :

(...) de grandioses funérailles de septième et de quarantième jour furent célébrées (quatre bœufs!). Les chasseurs se dépassèrent en miracles, en sorcelleries, et beaucoup de génies, beaucoup d'animaux, beaucoup de morts sous des formes humaines assistèrent à la fête pour rendre le suprême hommage au savoir et à l'expérience du vieux disparu. (180)

Si la description des funérailles de Koné Ibrahima expose en quelque sorte l'échéancier $\mathrm{du}$ rite funéraire chez les Malinkés, celles du cousin Lacina en explicitent la finalité escomptée, et celles de Balla leur confèrent leur dimension mythique (de croyance). Le lecteur saisit a contrario, et par association, ce dont Fama est spolié : le récit du rituel pour « rejoindre les ancêtres » et trouver « le calme intérieur ».

14 Loin d'être un simple épisode de l'histoire de Fama que la fiction crée pour la « dramatiser », le rite funéraire est non seulement amplement thématisé dans Les Soleils des indépendances mais c'est aussi un fait culturel dominant dans la culture des Malinkés.

De nombreux documents attestent en effet de ces rites funéraires qui permettent au mort d'accéder au repos éternel. Youssouf Cissé, par exemple, dans ses « Notes sur les sociétés de chasseurs malinké » (1964) (statut qui n'est pas étranger à celui de Fama), conclut ses observations sur le sens de la mort pour un Malinké :

Ainsi, la mort (...) représente pour lui [le chasseur malinké] le moment où son âme, enfin libérée de l'enveloppe qui la contenait, débarrassée du nyama dont elle s'était chargée au cours de son séjour dans le corps, purifiée, recouvre ses facultés et peut dès lors accéder à la vie éternelle ou à une nouvelle vie matérielle. Dans aucun de ces deux cas il n'y a de renouveau de l'âme. De ce fait, la mort, par les rites indispensables qu'elle suppose, serait plutôt délivrance de l'âme. (226)

Cette conception euphorique de la mort chez les Malinkés se manifeste à travers la présence de la musique et des chants au cours du rituel. Cissé Y. (idem) évoque la façon «dithyrambique » dont les louanges au mort sont chantées : «Le mort (...) est ensuite 
transporté sur un tyè kala au milieu de la cour pour les oraisons funèbres. (...) Quand tout est prêt, le sora, grand maitre de cérémonie, clame, en s'accompagnant de sa harpe, la louange des grands chasseurs du Manding » (210).

Ces «rites [funéraires] indispensables» de la culture malinké exigent en retour le respect strict des conventions. Il en est ainsi de la cérémonie du «lever du deuil » ou daga kari : " on ne procède au daga kari qu'au courant de la deuxième année survenant après le décès. Passé ce délai, l'Esprit du mort, sudya, vient hanter les vivants et exiger d'eux les funérailles indispensables au repos de son âme» (Cissé : 212).

Charles-Henry Pradelles De Latour le confirme dans son article intitulé «Les morts et leurs rites en Afrique » (1996):

Les rites funéraires se déroulent presque toujours en deux étapes au moins, qui correspondent d'une part aux rites d'enterrement, au cours desquels le mort se détache des vivants, d'autre part aux funérailles qui ont lieu quelques temps après et grâce auxquelles le mort, dépouillé des propriétés humaines, accède au statut d'ancêtre régulièrement vénéré ou à celui d'esprit occasionnellement invoqué.

La convergence entre le texte de fiction et le document est renforcée parce que c'est précisément dans ce sens que le rite est textualisé dans Les Soleils des indépendances. Le roman nous introduit pleinement dans la pensée mythique que Mircea Eliade explique dans Le Mythe de l'éternel retour. Il retient deux fondements principaux du mythe. Le premier est son caractère répétitif :

Tous les actes religieux sont supposés avoir été fondés par les dieux, héros civilisateurs ou ancêtres mythiques. Soit dit en passant, chez les "primitifs" non seulement les rituels ont leur modèle mythique, mais n'importe quelle action humaine acquiert son efficacité dans la mesure où elle répète exactement une action accomplie au commencement des temps par un dieu, un héros ou un ancêtre. (1969: 35)

Le second est celui de la distance entre temps mythique et temps profane. Selon lui, cette imitation des rites ancestraux abolit la distance temporelle qui sépare les descendants de leurs ancêtres. Autrement dit, durant le rituel, ces descendants quittent leur temps réel ou «temps profane" selon Eliade M., pour s'inscrire, par l'acte sacrificiel, dans le temps mythique, celui des commencements. D’où la vénération et le recueillement qui imprègnent les cérémonies rituelles :

Un sacrifice, par exemple, non seulement reproduit exactement le sacrifice initial révélé par un dieu ab origine, au commencement des temps, mais encore il a lieu en ce même moment mythique primordial ; en d'autres termes, tout sacrifice répète le sacrifice initial et coïncide avec lui. Tous les sacrifices sont accomplis au même instant mythique du commencement. (...) Par cette imitation, l'homme est projeté à l'époque mythique où les archétypes ont été pour la première fois révélés. (...) il y a abolition implicite du temps profane, de la durée, de l'"histoire", et celui qui reproduit le geste exemplaire se trouve ainsi transporté dans l'époque mythique où a eu lieu la révélation de ce geste exemplaire. (49-50)

21 Cette façon dont «le sacrificateur en pleine opération cérémonielle abandonne le monde profane des mortels et s'insère dans le monde divin des immortels » (idem) nous interpelle dans la mesure où celle-ci sera subvertie dans le roman de Kourouma, comme nous allons tenter de le démontrer. 


\section{De la réalité anthropologique à la fiction} rite funéraire chez les Malinkés.

\section{Fama, un mort sans sépulture}

Les trois personnages, Koné Ibrahima, Lancina et Balla, précédemment évoqués, ont eu droit à des funérailles, alors que leur rôle dans le roman est de moindre envergure par rapport à celui de Fama. Fama, pourtant personnage principal, prince à l'aura sacrée, dernier descendant des Doumbouya, en a été privé. La réduction du récit du rite funéraire à une série mécanique, routinière, de ses différentes phases simplement nommées, en une seule phrase (citée plus haut), provoque un effet de banalisation. Le narrateur relate, en effet, sous forme de ce que Genette (1972:130) appelle un "sommaire $»^{3}$, la succession des actes du rite funéraire, en faisant entendre, à travers l'article indéfini ( «un»), que Fama aura des funérailles comme n'importe quel Malinké : « Un Malinké était mort ». Fama, dernier descendant d'une grande dynastie, est noyé dans l'anonymat des Malinkés, et donc rabaissé, dévalorisé.

Ce qui contribue au renforcement de la banalisation du rite funéraire, dans ce passage, ce sont les trois points de suspension qui closent cette dernière phrase du roman. Cette suspension signifie l'inutilité de poursuivre le récit, sommaire de surcroît, dont la suite est connue du narrateur et du lecteur.

25 En effet, la banalisation-dépréciation est parachevée au niveau du signifiant: contrairement aux funérailles des autres personnages, narrées de façon à les individualiser - chacun d'eux a eu en quelque sorte "son rite» (comme signalé plus haut) -, celles de Fama s'écrivent sur la trace de celles des autres et dont le lecteur a déjà eu connaissance. Le début de la dernière phrase du roman, "Fama avait fini », fait écho à la première phrase du roman qui ouvre le récit des funérailles de Koné Ibrahima qui occupent tout le premier chapitre de la première partie depuis l'incipit : «Il y avait une semaine qu'avait fini dans la capitale, Koné Ibrahima, de race malinké ». L'effet routinier $\mathrm{du}$ rite est ainsi doublement signifiant : le récit peut se répéter à l'infini, comme le rite funéraire d'« un Malinké ». La logique mythique, qui voudrait que Fama ait «ses » funérailles, comme ceux de son rang, n'est pas respectée, pour lui, dans ce roman. Sa mort est banalisée, donc, désacralisée. Il n'en est pas de même pour celles des autres personnages.

\section{Les funérailles dans « le temps profane »}

Cependant, cela ne signifie pas que les funérailles des autres personnages, amplement narrées certes, soient situées dans le temps mythique. Si nous examinons de près le traitement réservé aux funérailles de Koné Ibrahima, Lacina et Balla, nous constatons que le caractère sacré du rituel subit des atteintes significatives. Le narrateur a recours aux sarcasmes, à l'humour, à la dérision, et surtout au langage scatologique. Il en est ainsi des funérailles de Koné Ibrahima qui ne se déroulent pas dans la sérénité exigée par la coutume. Le narrateur raconte que c'est à cette occasion que se réunissent les "vautours " et autres "hyènes ", qualifications méprisantes chez les Malinkés, pour désigner les «griots malinkés, les vieux Malinkés, ceux qui ne vendent plus parce que ruinés 
par les Indépendances (...) [et qui] "travaillent" tous dans les obsèques et les funérailles » (11). Par ailleurs, cette cérémonie a été perturbée par de violentes altercations entre Fama et certains convives :

Celui-ci [Fama] s'excitait, trépignait, maudissait : le fils de chien de Bamba montrait trop de virilité ! Il fallait le honnir, l'empoigner, le mordre. (...) Ils s'empoignèrent par les pans des boubous. Le griot s'éclipsa, le brouhaha s'intensifia; partout on se leva, s'accrocha, tira ; des pans de boubous craquèrent et se démêlèrent. (16)

Comme on peut le constater, le recueillement n'imprègne pas ces funérailles. Par ces invectives et ces empoignades, les convives sont restés dans le temps profane et le rite a perdu de sa sacralité. On peut ajouter, en se référant à Eliade M., que les participants à ce rite n'ont pas communié avec les ancêtres.

Aux funérailles de Balla le féticheur, au lieu de chanter ses louanges, comme dans le rite, «Bakary exerça toute sa verve pour exagérer les derniers exploits (entendez les dernières roueries) du vieux sorcier défunt" (180). L'intervention du narrateur, mise entre parenthèses, est capitale dans la mesure où elle éclaire le lecteur sur la nature des « exploits » du défunt dont il a pris soin de montrer, tout au long du roman, le caractère fourbe et mystificateur. De cette façon, Balla ne quitte pas le temps profane, même après sa mort.

Les funérailles de Lacina sont celles sur lesquelles le narrateur s'attarde le plus. Elles sont décrites explicitement comme des «funérailles pleinement réussies » (144) : «Un carnage, une ripaille viandée [qui] bouleversa toute la province" (139). "Des bousculades pareilles de bêtes et d'hommes pour du sang de funérailles" (145) sont "un (autre) signe incontestable» (145) de cette réussite :

(...) quand vint le partage de la viande rouge on procéda avec soin, (...) selon les coutumes (...). Puis les hommes rompirent le cercle, se dispersèrent, s'éloignèrent. Après eux, ils ne restèrent que les viscères et entrailles abandonnés à la marmaille. Et sans qu'on les appelât les enfants se ruèrent vers leur part comme des cordiers nains, ils tirèrent et promenèrent les intestins, s'enroulèrent dans les intestins, s'arrachèrent les boyaux. Et très rapidement, c'est-à-dire le temps de pousser des cris, de claquer des dents, la marmaille se dispersa et disparut (...). Les chiens furent admis après les enfants. Il ne restait plus rien, quelques pâtes d'excréments résultant du vidage des entrailles, beaucoup de mouches et du sang collé au sable.

On peut déjà constater que la dimension rituelle de la description, très présente à travers le strict respect de la hiérarchie dans le déroulement du cérémonial du " partage de la viande rouge » (les hommes, puis les enfants, et enfin les chiens), est en quelque sorte " profanée » par sa dimension scatologique. La désacralisation du rite au niveau du signifiant est confirmée au niveau du signifié narratif.

En effet, durant la cérémonie funèbre, «la piété aussi était partie; on lisait, priait d'un œil, l'autre caressait les boufs et les calebasses pleines de riz cuit" (141). Les convives demeurent, ici aussi, dans le temps profane des «boufs et [des] calebasses pleines de riz cuit ».

Nous pouvons dire dans ce cas qu'il y a immixtion du profane dans le sacré. Certes, les trois personnages ont eu leur rite funéraire, mais ce sont des rites entachés et dénués de sacralité. Cette série déceptive de la pensée mythique nous fait entrevoir l'hypothèse que ceux qui n'ont pas reçu le rite sont préservés. La vie de Fama - qui n'est donc pas tout à fait mort puisqu'il n'a pas reçu le rituel mortuaire - ne se prolongerait pas dans la 
logique mythique dégradée dans le texte, mais dans celle de l'écriture qui en a fait un mythe littéraire.

\section{Fama, héros tragique}

Dans le dernier chapitre du roman, Fama, quoique prince déchu, est doté d'une dimension imposante. Le chercheur Pierre Soubias, dans son article «Les Soleils des indépendances : le désenchantement » (2004) a déjà mis l'accent sur cet aspect :

Le récit de la mort de Fama achève de faire basculer notre rapport au personnage. L'ironie s'efface complètement au profit du pathétique, et le narrateur accompagne le prince déchu dans son agonie. Dans ce final tragique et grandiose, le fantasme du personnage n'est plus objet de dérision mais envahit tout l'espace du récit. (16)

Nous rejoignons sa vision dans le sens où Fama a suivi une évolution tout au long du roman, jusqu'à cette fin en apothéose. La noblesse de son attitude rappelle celle des grands personnages tragiques. L'écriture poétique qui imprègne ce chapitre et qui est dénuée de toute ironie ou dérision, contrairement au reste du roman, paraît significative d'une volonté du narrateur de restituer au prince sa grandeur au moment du dénouement tragique :

Fama sur un coursier blanc qui galope, trotte, sautille et caracole. Il est comblé, il est superbe. Louange au Miséricordieux! Mais Fama se retourne. Son escorte s'est évanouie. Où ont-ils disparu, mes suivants? proteste-t-il. Il est seul, il sent la solitude venir, elle assaille, pénètre dans son nez qui souffle un nuage de fumée, balaie les yeux, répand les larmes, vide le cœur, remplit les oreilles de la nausée jusqu'à ce que pointe et sorte la queue fuyante et le manque, Fama les pourchasse. Soudain un éclair explose, éparpille l'air, le ciel et la terre, et le coursier se cabre au bord du gouffre. Fama tremblote. Une prière. Tout s'arrange doux et calme, la douceur qui glisse, la femme qui console, et l'homme, et la rencontre d'un sous-bois frais et doux, et les sables menus et fins, et tout se fond et coule doucement et calmement, Fama coule, il veut tenter un petit effort. (195-196)

$\mathrm{Au}$ moment de la mort, Fama hallucine à travers une succession d'images hétéroclites. Le caractère poétique de ce passage apparaît à travers les rythmes binaire et ternaire qui font que les mots sont scandés. On le perçoit aussi dans la figure de l'énumération asyndétique des verbes (assaille, pénètre, balaie, répand, vide, remplit). Aussi voyons-nous les éléments naturels se déchaîner quand Fama approche de l'instant fatidique. Le feu, l'air et la terre l'accompagnent jusqu'au bord du gouffre qui l'attend, comme les plus grands personnages tragiques.

Un autre exemple illustre le destin tragique de Fama : la traversée « héroïque » de la frontière $e^{4}$. Cet épisode est très étudié par les critiques qui y voient un décalage total entre deux visions du monde. Celle de Fama, exclusivement tournée vers le passé glorieux de ses ancêtres qu'il doit honorer en poussant son dernier souffle sur sa terre natale, et celle de Bakary, des gardes frontaliers et de tout le peuple qui se presse aux festivités de la «Réconciliation nationale ». Cette dernière vision est attribuée à la propagande $\mathrm{du}$ «Parti unique " qui la dépeint comme «radieuse » pour ses fidèles adhérents. Le malentendu tragique qui oppose Fama au garde frontalier Vassoko illustre parfaitement l'absurdité des frontières qui ont fait éclater l'unité des tribus africaines et qui vont mener Fama à sa perte lorsqu'il tente de les traverser pour se rendre à Togobala, son village qui se trouve dans le pays voisin. C'est ce qu'explique Jacques Morel (cité par Puzin, 1987) : 
La tragédie, en effet, suppose l'acte. Le héros tragique entend s'affirmer dans le présent d'une action, fût-elle désespérée. Il n'existe que dans la mesure où il refuse d'être condamné seulement parce qu'il est homme, et veut mériter sa mort ou sa grâce par un acte libre. Il n'est jamais une victime, s'il consent parfois à être vaincu (...). De fait, le conflit tragique est toujours celui de l'humain et du divin, l'un tendant à rejoindre l'autre dans un dépassement héroïque qui lui permet de s'affirmer dans le refus, la soumission, ou l'adhésion enthousiaste. Crise décisive, au cours de laquelle le héros passe de l'existence à l'être, et se fixe à jamais dans une attitude exemplaire. ${ }^{5}(270)$

37 Ainsi, la traversée de la frontière est cet acte par lequel Fama refuse de se plier à l'incohérence du monde. Son acte de transgression qui se solde par sa mort fait de lui un héros tragique.

L'absurdité du monde à laquelle est confrontée Fama à «l'ère des Indépendances » est telle qu'explicitée par Pierre Brunel (2009) à propos de Camus :

Les cataclysmes de l'histoire moderne, le sentiment qu'a l'individu d'être jeté dans un monde incompréhensible et dont la représentation échoue par l'inadéquation du langage, telles sont les sources de la vision du monde profondément pessimiste que Camus appelle l'absurde.

39 Nous retrouvons la même vision de l'absurde dans l'échec du dialogue entre Fama et Vassoko, gardien intraitable des nouvelles frontières, qui s'obstine à lui refuser le passage sans la présentation d'une «carte d'identité ». Fama s'emmure dans la tradition des ancêtres et dans son langage, en déphasage avec les nouvelles exigences de "l'ère des Indépendances », comme cette « carte d'identité ». L'absurde ici, comme l'absurde camusien selon Pierre Brunel, consiste en ce que Fama, "confronté » à l' « irrationnel » du monde, et donc à la confusion, éprouve «ce désir éperdu de clarté dont l'appel résonne au plus profond de l'homme» (op .cit.).

\section{Fama : un mythe littéraire}

A travers cette étude, nous avons démontré la façon dont le texte s'est réapproprié des données culturelles afin de construire sa propre vision du devenir malinké postcolonial. En partant d'un contexte de la Côte d'Ivoire des années 1960, A. Kourouma a procédé à un travail d'écriture qui a produit une vision spécifique de cet univers, à travers le parcours du personnage de Fama. D'après notre démonstration, nous pensons pouvoir conclure sur l'accès de Fama au statut de mythe littéraire.

41 Le roman de Kourouma est travaillé par la dégradation des mythes de la tradition culturelle malinké à "l'ère des Indépendances»: le rite funéraire, l'un des «plus grands rites » malinkés, n'a plus le sens qu'il avait avant «la colonisation » et les «Indépendances ». Or, c'est à travers ce rite que le mort peut accéder à l'immortalité parmi les mânes des aïeux. Ce n'est pas cette immortalité qui a été choisie pour Fama, mais une immortalité littéraire. En ne le faisant pas tout à fait mourir selon la tradition, en laissant sa mort suspendue à trois points de suspension, en programmant le retour de l'excipit du roman à l'incipit (la fin est aussi commencement), Fama est " condamné » à un éternel recommencement, comme Sisyphe dans le mythe; le texte étant à la fois la montagne et le rocher. 
Il est pourtant paradoxal qu'un personnage individualisé (...) puisse se transformer en figure exemplaire et universelle, échappant à son créateur, s'émancipant de l'œuvre à laquelle il appartient pour devenir autonome et voyager d'un texte à l'autre. Tout personnage littéraire ne se transforme pas en mythe. Il lui faut, pour ce faire, être à la fois énigmatique et pluriel, chaque fois lui-même et pourtant autre dans toute œuvre nouvelle où il apparaît ; exprimer le système de représentation de l'époque où il voit le jour mais dépasser ces valeurs contingentes pour accéder à l'universel; et surtout être intégré à un récit archétypal, c'est-à-dire à une série de séquences dont l'association fait sens. (27)

L'association de Fama avec Sisyphe n'est pas la seule. Pierre Soubias, dans l'article cité précédemment, évoque sa similitude avec Don Quichotte de la Manche : «Le personnage de Fama possède une parenté évidente avec le Don Quichotte créé par Cervantès: même déphasage avec la réalité, même comique par conséquent et, pour finir, même impuissance ». Il cite lui-même l'ouvrage de Madeleine Borgomano, Ahmadou Kourouma, le guerrier griot (1998), dans lequel elle aurait mis en lumière "ce caractère "donquichottesque" de Fama » (13). Fama, dans son obstination à vénérer des ancêtres dont plus personne ne se souvient, à croire à sa mission de dernier descendant des Doumbouya que personne ne reconnaît, est, comme Don Quichotte, en rupture totale avec la réalité qui l'entoure.

Ainsi, Fama rejoint le panthéon des mythes littéraires. En tant que mythe littéraire, tenant à la fois de Sisyphe et de Don Quichotte, Fama incarne, dans le roman de Kourouma A., la condition à la fois tragique et absurde de l'homme africain de l'ère postcoloniale dont l'écrivain tourne en dérision le destin.

\section{BIBLIOGRAPHIE}

BRUNEL, Pierre, « Absurde », Encyclopaedie Universalis, 2009.

CISSÉ, Youssouf, « Notes sur les sociétés de chasseurs malinké », Journal de la Société des Africanistes, 1964, tome 34 fascicule 2, pp. 175-226. Disponible sur le site : [http://www.persee.fr/web/revues/ home/prescript/article/jafr_0037-9166_199166_1964_num_34_2_1383].

ELIADE, Mircéa, Le Mythe de l'éternel retour. Archétypes et répétition, Paris, Gallimard, coll. Folio Essais, 1969.

GENETTE, Gérard, Figures III, Paris, Seuil, 1972.

HUET-BRICHARD, Marie-Catherine, Littérature et mythe, Paris, Hachette Supérieur, coll. Contours littéraires, 2001.

KoURouma, Ahmadou, Les Soleils des indépendances, Paris, Seuil, 1970.

PRADELLES DE LATOUR, Charles-Henry, « Les morts et leurs rites en Afrique », L'Homme, 1996, tome 36 $\mathrm{n}^{\circ}$ 138, pp. 137-142. Disponible sur le site : [http://www.persee.fr/web/revues/home/prescript/ article/hom_0439-4216_1996_num_36_138_370080].

PUZIN, Claude, XVIIe siècle, Nathan, coll. Littérature, 1987. 
SOUBIAS, Pierre, «Les Soleils des indépendances : la magie du désenchantement », Notre Librairie. Revue des littératures $d u$ Sud, No 155 - 156, Identités littéraires, juillet - décembre 2004. Disponible sur le site : [http://www.institutfrancais.com/librairie/derniers/pdf/155-156_5.pdf].

\section{NOTES}

1. Nous nous référerons dans cet article à l'édition du Seuil, 1970.

2. L'éventail des recherches consacrées à ce roman, depuis sa première parution en 1968, est si vaste (14 400 résultats sur le net, au mois de mars 2013, pour la seule entrée « études de Les Soleils des indépendances ") qu'on ne citera ici que celles exploitées dans le sens de la problématique de cet article.

3. Pour G. Genette, un sommaire consiste en «la narration en quelques paragraphes ou quelques pages de plusieurs journées, mois ou années d'existence, sans détails d'actions ou de paroles » (1972:130).

4. Cet épisode intervient à la fin du roman, à partir de la page 188 .

5. MOREL, Jacques, La Tragédie, Armand Colin, 1964, pp. 7-8, cité par : PUZIN, Claude, XVIIe siècle, Nathan, coll. Littérature, 1987, p. 270.

\section{RÉSUMÉS}

Notre objectif est d'étudier dans Les Soleils des Indépendances ${ }^{1}$ d'Ahmadou Kourouma l'épisode constituant le dénouement tragique de ce roman: la mort de Fama. Notre lecture de cet événement se situe au confluent de deux interprétations: une mythification doublée d'une démythification de cette mort. En partant d'un détail, l'absence de rituel funéraire pour Fama, le personnage principal, nous nous interrogerons à propos de ce choix narratif. Nous analyserons la façon dont le rite funéraire a été subverti, et les incidences de ce traitement scripturaire sur la démythification puis la re-mythification de Fama.

Our aim in this paper is to study in The Suns of Independence, by Ahmadou Kourouma, the episode that constitutes the tragic end story of the novel: the death of Fama. Our reading of this event lies on crossing two interpretations: a doubled mythification of the demythification of this death. Starting from a detail, that is, the lack of a funerary ritual to Fama, who is the main character in this novel, we will question about this narrative choice. It is through the four steps that underly the ethnocritic approach that we analyze the way in which the funeral ritual was subverted in the text, and the incidences of this scriptural treatment on the demythification and the re-mythification of the death of Fama.

\section{INDEX}

Keywords : sub-Saharan Literature, mythical thought, literary Myth, funeral rite, mythification, demythification

Mots-clés : littérature subsaharienne, pensée mythique, mythe littéraire, rite funéraire, mythification, démythification 
AUTEUR

SABRINA ZOUAGUI

Université A. Mira - Bejaia 